\title{
Retardo del crecimiento fetal intrauterino
}

\author{
Jorge Eduardo Vélez A.*
}

RESUMEN: El retardo del crecimiento fetal intrauterino es el resultado de múltiples condiciones maternas y fetales. Su existencia se asocia con aumento de la morbimortalidad perinatal, lo que justifica intentar el diagnóstico precoz. Se describen dos tipos de retardo: Simétrico y Asimétrico. El retardo simétrico surge como consecuencia de patologías maternas preexistentes, o alteraciones que están presentes desde el comienzo de la gestación. El retardo asimétrico tiene como causas más frecuentes, las patologías que aparecen tardíamente en la gestación como la Preeclampsia eclampsia. El diagnóstico se basa primordialmente en datos de la historia y el examen clínico, utilizando como prueba confirmatoria el ultrasonido. El tratamiento ideal es la finalización del embarazo una vez se han analizado factores tales como la edad gestacional, la madurez fetal y la posible causa determinante.

PALABRAS CLAVES: Retardo del crecimiento intrauterino, alto riesgo fetal.

SUMMARY: The intrauterine growth retardation is the result of multiple mother and fetal conditions. It has been related to the perinatal morbimortality increase which justifies to attempt at its early diagnostic. Two type of retardation have been described: symmetric and asymmetric. The symmetric retardation is the consequence of preexistent mother pathologies or alterations at the initiation of the pregnancy process. The asymmetric retardation is caused by pathologies that appear late on the pregnancy such as the preeclampsia eclampsia. The diagnostic is mainly based on the history data and the clinic examination by using the ultrasound as the confirmatory probe. The ideal treatment is the end of pregnancy once factors such as the gestational age, fetal maturity and the possible cause have been analyzed.

KEY WORDS: Intrauterine growth retardation, high fetal risk.

\section{Introducción}

El retardo del crecimiento fetal intrauterino es sin lugar a dudas una condición asociada con aumento de la morbilidad y mortalidad perinatales. Su diagnóstico se consigue con un alto grado de sospecha y una cuidadosa evaluación del comportamiento de la ganancia de peso durante la gestación y evolución de la altura uterina, en asociación con el empleo de la ecografía como método diagnóstico de mayor confiabilidad.

\section{Definición}

Todos aquellos recién nacidos con peso al nacer inferior al esperado para la edad gestacional se consideran como productos con retardo del crecimiento.

Dicha definición contrasta con la de Bajo Peso al Nacer, que se define como peso inferior a 2500 gramos, ya que muchos productos presentan pesos superiores a 2500 gramos y se les clasifica como retardados en el crecimiento en vista de que no alcanzaron todo su potencial de desarrollo en cuanto a peso se refiere.

La mejor definición de R.C.I.U. sería entonces todo peso al nacer menor que el percentil 10 de normalidad para una edad gestacional determinada (1-5).

Gineco - Obstetra. Profesor Asistente. Jefe Sección Obstétrica. Facultad de Medicina. Universidad de Caldas.
En la elaboración de una curva de peso deben tenerse en cuenta factores tales como: Sexo fetal, raza, nivel sobre el mar, e incluir para el cálculo de los percentiles solamente productos de embarazadas de bajo riesgo y de nivel socioeconómico adecuado. Estas consideraciones permitirán disponer de curvas de peso que faciliten el diagnóstico real de la frecuencia de retardo del crecimiento en una población determinada. $(4,18)$.

Las definiciones mencionadas consideran al recién nacido, en el cual lógicamente podemos determinar con facilidad el peso y establecer el diagnóstico probable. La situación obviamente resulta más complicada cuando se refiere al feto, pues la sospecha diagnóstica se basa en medidas fetales determinadas por ecografía, cuyos rangos de variabilidad son bien conocidos $(4,13,17)$.

Como ya se mencionó otro concepto importante a tener en cuenta, son las grandes variaciones en el peso fetal asociadas con factores tales como la raza, el nivel sobre el mar en el que habita la madre, y el sexo fetal entre otros. A manera de ejemplo, el peso promedio al nacer de los indios Cheyenne de los Estados Unidos es de 3700 gr. mientras el de los recién nacidos de la tribu Lummy en Nueva Guinea es sólo de 2400 gr. (1).

Debe manejarse entonces la idea de la existencia de productos "PEQUEÑOS NORMALES" que se comportan de manera normal durante el embarazo y el parto y su desarrollo post-natal transcurre también normalmente. 


\section{Morbimortalidad}

La tasa de mortalidad perinatal es 5 a 10 veces superior, que la que ocurre en productos de peso adecuado; asociándose también con un incremento del riesgo de asfixia intraparto. El feto con retardo del crecimiento tiene entonces un aumento evidente del riesgo de muerte intrauterina y muerte neonatal temprana, como también de complicaciones en el período neonatal o en su desarrollo neurológico posterior, en los que sobreviven. (1, 5-6 $9,25)$. Desafortunadamente, un porcentaje importante de casos de R.C.I.U., escapan al diagnóstico y éste sólo se establece en el momento del parto, o se presume en casos de muerte intrauterina de causa indeterminada.

Al momento del nacimiento el producto con R.C.I.U. muestra signos de compromiso a nivel de sus tejidos blandos. La piel es delgada y existe poca grasa subcutánea. El abdomen es escafoide, las costillas prominentes y la masa muscular a nivel de los brazos, nalgas y muslos está disminuida. El cordón umbilical es flácido, delgado $\mathrm{y}$ frecuentemente teñido de meconio y tanto el peso fetal como el placentario están por debajo del percentil 10 de normalidad, identificándose la mayoría de las veces, una circunferencia cefálica mayor que la abdominal. En contraste el niño pequeño para la edad gestacional sano, se presenta con un desarrollo simétrico de la cabeza y el abdomen; y tiene una cantidad normal de grasa subcutánea $(10,21)$.

Mientras que el niño pequeño normal pocas veces tiene problemas, el producto con retardo del crecimiento desarrolla frecuentemente complicaciones, entre las que se destacan:

1.Complicaciones relacionadas con asfixia perinatal y acidosis: Circulación fetal persistente, aspiración de meconio, encefalopatía hipóxica isquémica.

2.Alteraciones metabólicas: Hipoglicemia, hipocalcemia, hiperviscosidad, hiperglicemia e hipotermia.

3. Complicaciones relacionadas con la causa específica del retardo del crecimiento: Infecciones, malformaciones, anormalidades cromosómicas $(3,10)$.

\section{Reseña histórica}

En 1947 McBurney llamó la atención sobre una situación definida por él como "El niño de término desnutrido", para referirse a productos de término con peso inadecuado. En 1960, Gruenwald introdujo el concepto de insuficiencia placentaria, como una explicación a muchos casos de R.C.I.U. En el mismo año la Doctora Lubchenco publicó sus tablas de peso al nacer de acuerdo con la edad gestacional, identificando condiciones de riesgo de acuerdo con peso al nacer. En la actualidad se ha descifrado en gran parte la fisiopatología del retardo del crecimiento, identificándose factores de riesgo y condiciones predisponentes asociados con la patología $(12,21)$.

\section{Etiología}

De acuerdo con Gruenwald, pueden clasificarse en tres categorías las causas de retardo del crecimiento: Fetales, maternas y placentarias.

\section{Causas fetales}

Anomalías congénitas, trisomías, infecciones intrauterinas (TORSCH), Embarazo múltiple.

Algunos estudios parecen sugerir una mayor frecuencia de retardo del crecimiento asociada con el parto pretérmino (14).

Una de las complicaciones del embarazo post-término es también el retardo del crecimiento fetal.

\section{Causas maternas}

Enfermedades cardiopulmonares, trastornos hipertensivos, anemia severa, malnutrición, adicción a drogas, tabaquismo (20).

\section{Causas placentarias}

Placenta pequeña, placenta circunvalata, inserción anormal del cordón, inserción placentaria anormal, infartos placentarios, abruptio placentae.

En muchos casos, no se logra establecer la causa definida del síndrome a pesar de estudios cuidadosos.

\section{Fisiopatología}

Son factores reguladores del crecimiento fetal: Substrato materno adecuado, flujo sanguíneo, útero placentario adecuado, placenta normal y feto normal.

Cualquier trastorno a nivel de estos factores determinará alteraciones del crecimiento fetal intrauterino, cuya gravedad estará relacionada con la magnitud de la alteración, y el período de la gestación es el que ocurre.

Se han definido dos tipos de R.C.I.U.: Simétrico y asimétrico.

El retardo del crecimiento simétrico se caracteriza por una disminución de todas las medidas antropométricas fetales y supone la existencia de factores causales desde el comienzo de la gestación. Son ejemplos de factores etiológicos del retardo simétrico, las trisomías y las infecciones virales del primer trimestre de la gestación, como situaciones que existen desde el comienzo mismo de la gestación; y la enfermedad hipertensiva crónica de la madre como ejemplo de una patología que ya existe antes del comienzo de la gestación, de origen a trastornos del crecimiento fetal relacionados con la alteración del flujo uteroplacentario.

En el retardo del crecimiento asimétrico la alteración del crecimiento se manifiesta sólo en algunos parámetros fetales, y el ejemplo clásico de la patología es la gestación afectada por hipertensión inducida por el embarazo (Preeclampsia - Eclampsia), situación en la cual el feto venía desarrollándose de manera normal, y al establecerse la patología, se alteran parámetros del crecimiento fetal, que tienen períodos críticos del desarrollo en determinadas etapas de la gestación. En el caso del retardo asimétrico, la alteración va a manifestarse en una detención del aumento del perímetro abdominal, mientras que el perímetro cefálico puede no comprometerse $(1,3,5,19)$.

\section{Diagnóstico}

El principal elemento diagnóstico del retardo del crecimiento es la sospecha clínica basada en el análisis 
juicioso de los antecedentes médicos y obstétricos y en los hallazgos del control prenatal.

\section{Métodos diagnósticos}

Historia clínica.

Historia obstétrica.

Ganancia de peso.

Evolución de la altura uterina.

Ultrasonografía.

Antecedentes maternos, como hipertensión arterial, enfermedades cardiopulmonares crónicas, diabetes, tabaquismo y desnutrición, pueden considerarse factores predisponentes. El antecedente de retardo del crecimiento en gestaciones previas, y la presencia de embarazo gemelar en el embarazo actual se consideran también factores asociados.

Todas las pacientes con factores de riesgo, o aquellas con progreso anormal de la altura uterina o ganancia de peso inadecuada, deben remitirse para seguimiento ecográfico. Una herramienta útil durante el control prenatal son las curvas de incremento de peso materno y de evolución de la altura uterina según la edad gestacional, propuestas por el Centro Latinoamericano de Perinatología (CLAP), las cuales nos permiten la identificación de las alteraciones en la ganancia de peso y en el progreso de la altura uterina.

\section{Tabla 1}

\section{INDICACIONES PARA SEGUIMIENTO ECOGRAFICO}

\footnotetext{
- Antecedente de productos de bajo peso para la edad gestacional.

- Embarazo múltiple.

- Poca ganancia de peso.

- Crecimiento lento de la altura uterina.

- Presencia de patologías que alteren el crecimiento fetal.

- Tabaquismo.

- Drogadicción.
}

\section{Diagnóstico por ultrasonido}

La ecografía bidimensional se considera el método más adecuado para el diagnóstico del R.C.I.U.

Las mediciones ecográficas que permiten diagnosticar el retardo del crecimiento son: Diámetro biparietal, perímetro craneano, perímetro abdominal, relación perímetro craneano/perímetro abdominal, fémur, radio fémur/ abdomen (1, 3-4, 6, 13, 24).

Existen tablas para identificar la normalidad de las medidas fetales de acuerdo con la edad gestacional, y cuando el dato estimado está por debajo del percentil 5 de normalidad para dicha edad gestacional, se considera que la medida es anormal. En nuestro medio se utilizan las tablas propuestas por Fescina en el Centro Latinoamericano de Perinatología (4).
La relación perímetro craneano/perímetro abdominal va disminuyendo progresivamente a lo largo de la gestación hasta ser igual a 1 para la semana 37 , debido a que la velocidad de crecimiento del cráneo se hace más lenta, mientras el crecimiento del abdomen continúa en aumento.

En síntesis, los elementos para el diagnóstico del retardo del crecimiento y sus diferentes tipos son los siguientes:

En el retardo simétrico, todas las determinaciones: DBP, perímetro craneano y perímetro abdominal están por debajo de los percentiles de normalidad. La relación perímetro craneano /perímetro abdominal será normal a lo largo de la gestación.

En el retardo asimétrico, el perímetro abdominal empieza a alejarse de lo normal, conservándose el diámetro biparietal y el perímetro craneano dentro de percentiles normales. En este caso, la relación perímetro craneano/ perímetro abdominal será alta para la edad gestacional, pues como se explica, mientras el perímetro craneano se conserva, el abdominal se compromete.

Debe destacarse sin embargo, que en muchos casos el diagnóstico preciso del tipo de retardo puede dificultarse en razón a que en muchas situaciones pueden darse condiciones superpuestas que da origen a formas de retardo del crecimiento con componentes del tipo simétrico y asimétrico (15).

Otros hallazgos ecográficos como una placenta grado III, y un líquido amniótico disminuido o ausente permiten confirmar el diagnóstico y son indicaciones para finalizar la gestación.

El Ultrasonido Doppler, una de las más recientes tecnologías disponibles en perinatología, aparece en la actualidad como un método con posibilidades diagnósticas del retardo del crecimiento intrauterino. Desafortunadamente su alto costo y las controversias existentes con respecto a su capacidad diagnóstica, no permiten recomendarlo todavía como elemento diagnóstico de rutina $(1,22)$.

\section{Normas de manejo}

1. En casos de gestación de 37 semanas o más, la conducta correcta es la finalización del embarazo, mediante inducción del parto o cesárea de acuerdo con las condiciones obstétricas. Debe incrementarse al máximo la vigilancia fetal durante el trabajo de parto y el parto, por el riesgo de sufrimiento fetal presente en estos casos.

2. En embarazos con edad gestacional menor de 37 semanas, la continuación de la gestación dependerá del estado materno y fetal; cuando estos lo permitan, el embarazo se continuará hasta la semana 37 o hasta cuando se compruebe la madurez pulmonar fetal.

3. Cuando se decide continuar la gestación, deben emplearse métodos de vigilancia fetal tales como: Monitorización fetal clínica y electrónica, seguimiento ecográfico, perfil biofísico fetal. Se intentará el manejo de patologías maternas susceptibles de tratamiento (hipertensión crónica, enfermedades cardiopulmonares etc.), recomendándose además, reposo en cama, dieta adecuada y supresión del cigarrillo. 
4. Se ha indicado el uso de beta miméticos a bajas dosis (2.5 a $5 \mathrm{mg}$.) como medio para mejorar la perfusión uteroplacentaria. Con el mismo fin se ha propuesto también el uso de la aspirina en bajas dosis (100 mg./día), invocando su efecto estimulante de la producción de prostaciclina $(11,23)$.

5. La hiperoxigenación materna se ha sugerido como una estrategia para mejorar la disminución de la perfusión uteroplacentaria y corregir la acidosis fetal existente en casos de retardo del crecimiento severo. Sin embargo esta terapia debe considerarse en etapa experimental por el momento (16).
6. En aquellas situaciones de retardo del crecimiento simétrico severo, deben agotarse todos los medios disponibles para descartar la posibilidad de problemas cromosómicos fetales responsables de la patología, caso en el cual, el manejo obviamente variaría. De ser posible debe remitirse la paciente a instituciones capacitadas en el diagnóstico genético prenatal en las que sea posible realizar ecografía de IV nivel y procedimientos especializados como cordocentesis o amniocentesis diagnóstica.

La Tabla 2 resume los pasos necesarios para el diagnóstico y el manejo del retardo del crecimiento.

Tabla 2

DIAGNOSTICO Y MANEJO DEL RETARDO DEL CRECIMIENTO INTRAUTERINO

\section{FLUJOGRAMA}

SOSPECHA DIAGNOSTICA: Antecedentes médicos y obstétricos

Ganancia de peso inadecuada

Evolución anormal de la altura uterina

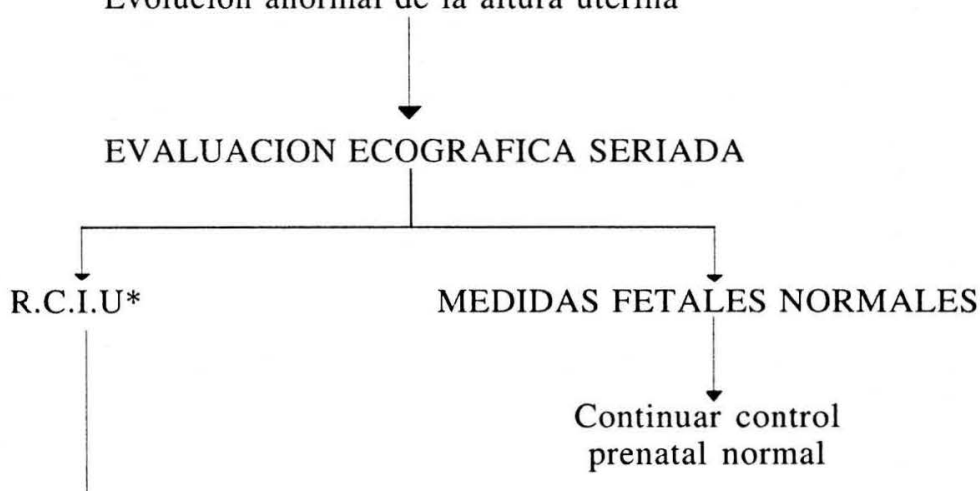

INTENTAR CLASIFICARLO

Simétrico-Asimétrico

Edad gestacional:

37 semanas o más

DESEMBARAZAR

Menor de 37 semanas

DETERMINAR MADUREZ PULMONAR

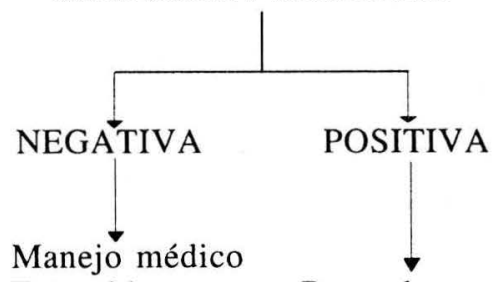

Esteroides

Desembarazar

Desembarazar cuando se compruebe madurez pulmonar o en caso de sufrimiento fetal.

R.C.I.U: Retardo del crecimiento fetal. 


\section{BIBLIOGRAFIA}

1. Arias F. Practical Guide to High-Risk Pregnancy and Delivery. Second edition. Mosby Year Book. 1993.

2. Pritchard J., Macdonald P., Williams. Obstetrics. Appleton-CenturyCrofts. New York. Sixteenth Edition. 1980; 942-949.

3. Main E. Intrauterine Growth Retardation, in: Arias F, High-Risk Pregnancy and Delivery. The C.V. Mosby Company. 1984; 148-172.

4. Fescina R. Evaluación del crecimiento intrauterino. Introducción a la Ultrasonografía en Perinatología. Publicación científica No. 948. Centro Latinoamericano de Perinatología y Desarrollo Humano. 1984.

5. Eden R., Boehm F. Assessment and Care of the Fetus. Physiological, Clinical, and Medicolegal Principles. Appleton - Lange. 1990.

6. Creasy R., Resnik R. Maternal-Fetal Medicine: Principles and Practice. W.B. Saunders Company. Second edition. 1989.

7. Queenan J. Atención del Embarazo de Alto Riesgo. Manual moderno. Segunda edición. 1987.

8. Rey H. El recién nacido Latinoamericano. Atención primaria y secundaria según riesgo. Impresora Feriva. Cali. 1986.

9. Iffy L., Kaminetzky H. Obstetricia y Perinatología. Principios y práctica. Editorial Médica Panamericana. 1985.

10. Cifuentes R. Obstetricia de Alto Riesgo. Talleres gráficos imprenta departamental. Cali, Colombia. 1987.

11. Barton J., Baha MS. Low-Dose Aspirin to Improve Perinatal Outcome. Clinical Obstetrics and Gynecology. 1991; 34(2): 251-261.

12. Mann L., Thomasson J., Widness J. Identification and Management of Intrauterine Growth Retardation. In: Zuspan F., Christian C. Controversy in Obstetrics and Gynecology-III. W.B. Saunders Company. 1983.

13. Deter R., Hadlock F., Harrist R. Evaluación del crecimiento fetal normal y detección del retardo del crecimiento intrauterino. En: Callen P. Ultrasonografía en Obstetricia y Ginecología. Edit. Panamericana. Buenos Aires. 1985; 119-148.
14. Ott $\mathrm{W}$. Intrauterine growth retardation and preterm delivery. American J. Obstet. Gynecol. 1993; 168: 1710-1717.

15. Lin Ch., Su S., River P. Comparison of associated factors and perinatal outcome between symmetric and asymmetric fetal intrauterine growth retardation. Am. J. Obstet. Gynecol. 1991; 164: 1535-1542.

16. Battaglia C., Artini P., Galli P. Maternal hyperoxygenation in the treatment of intrauterine growth retardation. Am. J. Obstet. Gynecol. 1992; 167: 430-435.

17. Madrazo B. Retardo del crecimiento intrauterino y el feto macrosómico. En: Reynes C., Stoopen M. Ultrasonografía en obstetricia. Editorial Interamericana. México, D.E. 1992; 107-112.

18. Goldenberg R., Cutter G., Hoffman H., Foster J. Intrauterine growth retardation: Standards for diagnosis. Am. J. Obstet. Gynecol. 1989; 161: 271-277.

19. Schwarcz R., Duverges C., Díaz A. Obstetricia. Editorial El Ateneo. Cuarta edición. 1987.

20. Salud Perinatal. Tabaquismo y Embarazo: Hay que ayudar a parar. Boletín del Centro Latinoamericano de Perinatología y Desarrollo Humano (CLAP). Vol. 2 No. 71987.

21. Danforth D. Tratado de Obstetricia y Ginecología. Editorial Panamericana. Cuarta edición. 1988; 671-674.

22. Fleischer A., Romero R., Manning F. The Principles and Practice of Ultrasonography in Obstetrics and Gynecology. Prentice-Hall International Inc. Fourth edition. 1991.

23. Barton J., Sibai B. Low-Dose Aspirin to Improve Perinatal Outcome. Clinical Obstetrics and Gynecology. 1991; 34(2): 251-261.

24. Mintz M., Landon M. Sonographic Diagnosis of Fetal Growth Disorders. Clinical Obstetrics and Gynecology Vol. 31 No. 1.

25. Hacker N., Moore J. Essentials of Obstetrics and Gynecology. W.B. Saunders Company. 1986. 